\title{
Automatic trinocular 3D reconstruction of coronary artery centerlines from rotational X-ray angiography
}

\author{
C. Blondel, R. Vaillant ${ }^{\mathrm{b}}$, F. Devernay ${ }^{\mathrm{a}}$, G. Malandain ${ }^{\mathrm{a}}$, N. Ayache ${ }^{\mathrm{a}}$ \\ ${ }^{a}$ INRIA, 2004 rte des Lucioles, BP 93, 06902 SOPHIA ANTIPOLIS, France \\ ${ }^{\mathrm{b}}$ GEMSE, 283 rue de la Minière, 78530 BUC, France
}

\begin{abstract}
We present a method for fully automatic 3D reconstruction of coronary artery centerlines using three X-ray angiogram projections from a single rotating monoplane acquisition. The reconstruction method consists of three steps: (1) filtering and segmenting the images using a multiscale analysis, (2) matching points in two of the segmented images using the information from the third image, and (3) reconstructing in 3D the matched points. This method needs good calibration of the system geometry and requires breatheld acquisitions. The final algorithm is formulated as an energy minimization problem that we solve using dynamic programming optimization. This method provides a fast and automatic way to compute 3D models of vessels centerlines. It has been applied to both phantoms, for validation purposes, and patient data sets.
\end{abstract}

Keywords: coronary angiography, 3D reconstruction, matching

\section{Introduction}

$\mathrm{X}$-ray angiography is the most frequently used imaging modality to diagnose coronary artery diseases and to assess their severity. Traditionally, this assessment is performed directly from the angiograms, and thus, can suffer from viewpoint orientation dependence and from lack of precision of quantitative measures due to magnification factor uncertainty. Three dimensional (3D) reconstruction of the coronary arteries from the angiograms would lead to higher accuracy and reproducibility in the diagnosis and to better precision in the quantification of the severity of the diseases ([1], [2]). Reconstructing the coronary artery centerlines provides geometrical and topological information that is necessary to compute the optimal orientation of the imager for stenosis characterization ([3]) or to give an initial point to the computation of the deformation field of the vessels along the cardiac cycle ([4], [5]).

\section{Methodology}

\subsection{Images acquisition and segmentation}

Automatic reconstruction methods using only two images ([2], [5]) have not been shown to be sufficiently reliable for a wide clinical application. Because we are interested in an automatic procedure, we have chosen to use three images. The acquisition routine we used 
provides a way to obtain them, performing one run with a monoplane system. An angiogram sequence is acquired with a single $\mathrm{C}$-arm, which rotates around the patient. We determine the acquisition geometry from system calibration using a helical phantom. The rotational acquisition is performed with a zero Cranial/Caudal angle and with the Left Anterior Oblique (LAO)/Right Anterior Oblique (RAO) angle varying from 90 degrees RAO to 90 degrees LAO. Three images of the same cardiac phase are selected from the angiogram sequence. Depending on the rotation speed and on the heart rate of the patient, the LAO/RAO angle between two successive selected images varies between 25 degrees and 40 degrees.

The images are segmented using multiscale analysis and local maxima extraction ([6]). A set of scales is selected, taking into account the diameters of the coronary arteries we want to extract. For each of the selected scales, the image is convoluted by a gaussian kernel with size corresponding to the scale. At each image point, we compute the intensity and the direction of the response of a rectilinear structure detector based on the eigenvalues of the Hessian matrix of the image greyscale values. For a given point, the intensity encodes the likelihood of belonging to a rectilinear structure and the direction encodes a tangent vector to this rectilinear structure. After iterating at each scale, we compile a multiscale analysis that summarizes, for each image point, the scale that corresponds to the largest response, and the corresponding response value and tangent angle ([7]). We store these values as scale, response, and tangent maps.

To segment the vessels, we then apply a hysteresis thresholding to the response maps. Hysteresis thresholding of a map retains connected components with all points that have values above a low threshold and with at least one point with a value above a high threshold. The two thresholds are computed as constant quantile values (at 90\% and 98\%) of the histogram of response maps generated from the three images. Finally, local maxima extraction of the thresholded response maps leads to a segmentation of the coronary artery centerlines in the angiograms. The segmentation's output is a list of linked points we call "chains". This process is fully automatic. Although the general quality of the segmentation is pretty good, some discrepancies between the segmentation and the real vessel tree may occur. A first difficulty is that vessels can be broken into several disconnected components and that discontinuities may occur at bifurcation points. A second difficulty is that two distinct vessels can superimpose in the angiogram and be detected as one single vessel. Consequently, the matching process has to be robust with respect to the segmentation it uses as input.

\subsection{Potential matching points computation}

Potential matching points are determined in the following manner: for each point $p^{1}$, we compute the corresponding epipolar line $l^{12}$ in the second image and compute its intersections with the second image's segmentation. We note $\left\{p^{12}{ }_{k}\right\}_{k}$ this set of all points that potentially match with $p^{1}$. We call these points "candidates". Typically, in the experiments we conduced, the number of candidates was about 5 in average and ranged from 0 to 15 .

Once all the candidate matching points are determined, we assign them a score based on the intensity and direction information the multiscale analysis provides. For each value of $k$, we can compute, using stereoscopic reconstruction, $r^{12}{ }_{k}$ the corresponding reconstructed 
3D point and $p^{123}{ }_{k}$ the corresponding reprojected point in the third image (see figure 1). We now can assign a score $s^{123}{ }_{k}$ to any choice of a $k$ value, using the values of multiscale analysis at position $p^{123}{ }_{k}$ in the third image. Natural choices for this score can be the multiscale intensity at these positions or the angular closeness between computed and predicted tangents ([8], [9]). Finally, we use a multiplicative combination of these scores because the correct matching points should give simultaneously large multiscale intensity values and good correspondences between computed and predicted tangents.

We iterate this process for every point of every chain of the first image's segmentation. We now index chains numbers by $i$ and points numbers by $j$ (for instance, $p^{12}{ }_{i, j, k}$ denotes the $k^{\text {th }}$ candidate of the $j^{\text {th }}$ point of the $i^{\text {th }}$ chain in the first image's segmentation).

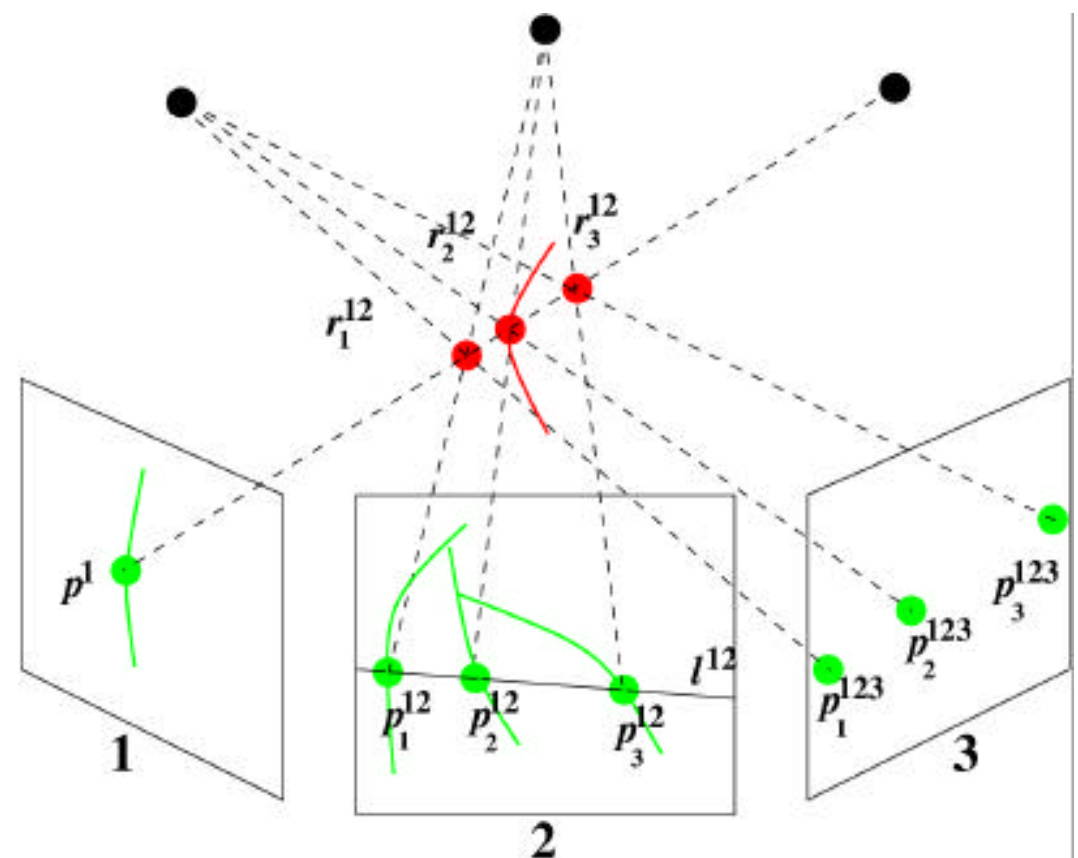

Figure 1: $p^{1}$ is in the segmentation of image $1,\left\{p^{12}{ }_{\mathrm{k}}\right\}_{\mathrm{k}}$ are its matching candidates in image $2,\left\{\mathrm{r}^{12}\right\}_{\mathrm{k}}$ are the associated reconstructed points, and $\left\{p^{123}\right\}_{\mathrm{k}}$ are the associated reprojected points in image 3

\subsection{Selection algorithm}

When attempting to match the points, two approaches are possible: point to point matching and chain to chain matching. For fixed given values of $i$ and $j$, point to point matching would consist in selecting the value $k$, and thus the point $p^{12}{ }_{i, j, k}$, that gives the best score $s_{i, j, k}^{123}$. For a fixed given value of $i$, chain to chain matching would consist in selecting the values $\left\{k_{j}\right\}_{j}$ that produce the connected set of candidates among the set $\left\{p^{12}{ }_{i, j, k}\right\}_{j, k}$, with the best sum of scores $\Sigma_{i} s_{i, j, k}^{123}$. We do not retain these two approaches because of the following arguments. The point to point matching algorithm does not take into account the consistent geometrical behaviour of neighbouring points and thus gives noisy and geometrically incoherent reconstructions. The chain to chain matching algorithm assumes implicitly that the segmentations have no cuts and that a chain in the 
first image represents only one artery. These hypotheses are necessary to ensure connexity of the matched chain in the second image and of the reprojected chain in the third image. Violating these hypotheses makes the algorithm produce false reconstructed vessels.

We observe that, in most cases (except when vessels are superimposed), two neighbouring points in an angiogram are real neighbours in $3 \mathrm{D}$, and thus should be matched and reprojected as neighbours in the other angiograms. We see that, relatively to the former remark, point to point matching is underconstrained and chain to chain matching is overconstrained. We also want to allow a point not to be matched if it has no candidate or if all the candidates infer only a small score positive contribution compared to the geometrical incoherence they induce. Taking all of this into account, we build a semi-local energy formulation of the matching problem. For a given value of $i$, we compute the sequence of candidates indices $K_{i}^{123}=\left(k^{123}{ }_{i, 1}, \ldots, k_{i, J}^{123}\right)$ that minimizes:

$E_{i}^{123}(K)=-\Sigma_{j} s_{i, j, k_{j}}^{123}+\alpha \Sigma_{j} T\left(p_{i, j, k_{j}}^{12}, p^{12}{ }_{i, j, k_{j+1}}\right)$ where $T\left(p_{i, j, k_{j}}^{12}, p_{i, j, k_{j+1}}^{12}\right)$ denotes the geometrical penalty between two successive matched points. We now allow the case $k=0$, which encodes the fact that we choose not to match the point with any candidate. We take as a convention $s_{i, j, 0}^{123}=0$ and $T\left(p_{i, j, k_{j}}^{12}, p_{i, j, k_{j+1}}^{12}\right)=0$ if $k_{j}=0$ or $k_{j+1}=0$. This implicitly means that a point that is not matched has no positive score contribution nor negative geometrical penalty contribution. $T$ can be, for instance, the $2 \mathrm{D}$ distance in the second image between the matched points of two successive points in the first image: $T\left(p_{i, j, k_{j}}^{12}, p^{12}{ }_{i, j, k_{j+1}}\right)=\left\|p_{i, j, k_{j}}^{12} p_{i, j, k_{j+1}}^{12}\right\|$.

We remark that if the geometrical penalty is above a threshold, it indicates a change in the $3 \mathrm{D}$ connected component we match. As soon as we have identified a 3D discontinuity in the matching, the penalty must remain constant. Thus, we give a high threshold to the penalty $T$, to make it more robust and not to privilege close disconnected $3 \mathrm{D}$ components with respect to far disconnected 3D components.

We solve this global recursively-defined minimization problem using a dynamic programming method.

\subsection{Reconstruction algorithm symmetrization}

The former algorithm is asymmetric: it depends fundamentally on the ordering of the three images. We can symmetrize the algorithm by applying it to the six possible orderings and gathering the six different reconstructions. It also allows recovering vessel parts that could not correctly be reconstructed using only one specific ordering because of the occlusion and depth effects.

To produce the algorithm output, we gather, for each ordering, the matched points from all the chains and then gather the six reconstructions obtained by reordering the images.

\section{Results}

We applied the algorithm to a physical coronary artery tree phantom and to data sets acquired from patients who held their breath. We show projected views of the resulting $3 \mathrm{D}$ reconstructed centerlines for a patient data set in figure 2 . The images are $512^{2} \mathrm{x} 8 \mathrm{bpp}$. Typical running time of our method on a $333 \mathrm{MHz}$ Sun Ultra 10 workstation is 14 seconds for thesegmentation process and 6 seconds for the matching process. 

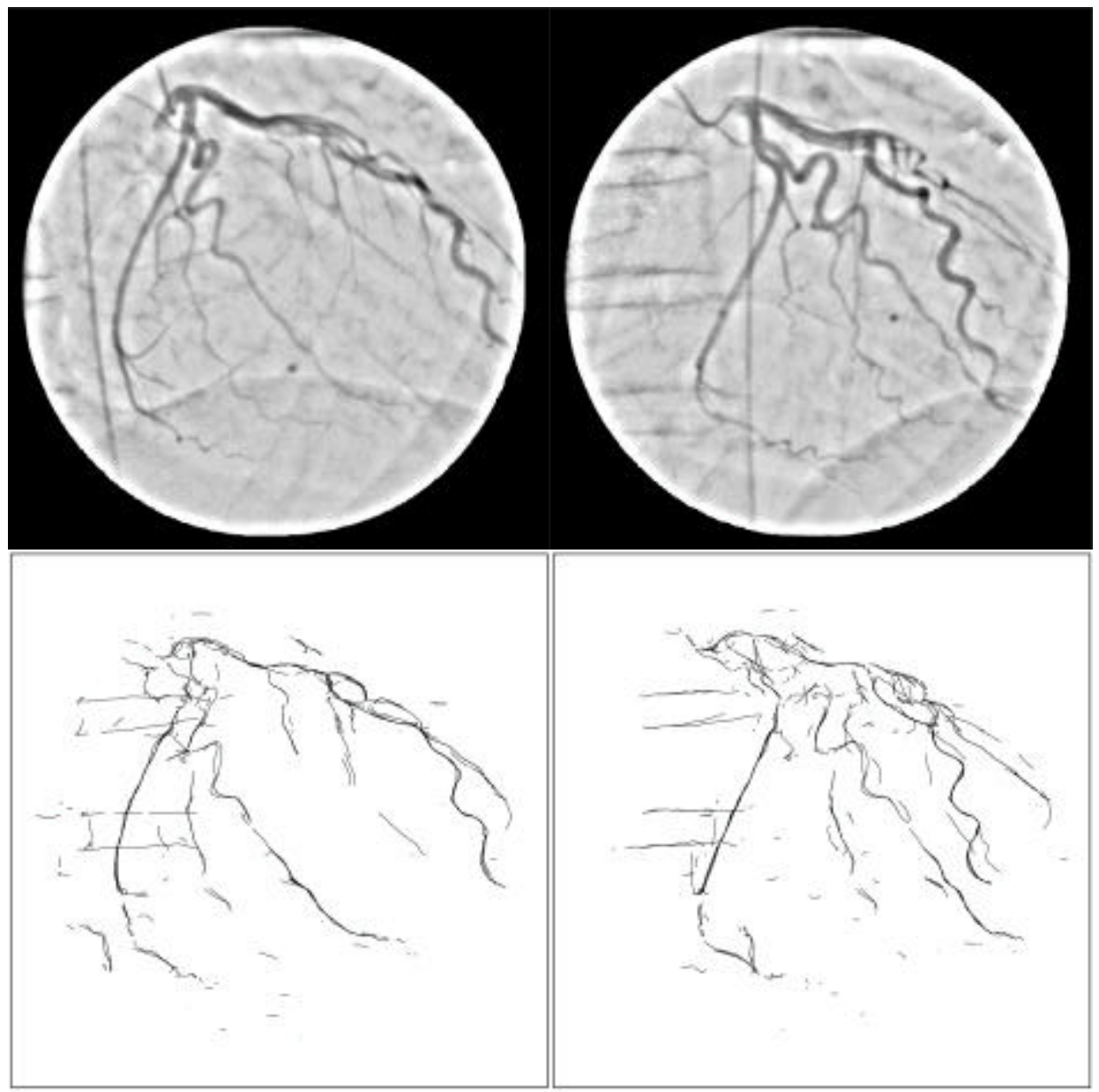

Figure 2: top images show two views, from a patient data set, bottom images show the corresponding reprojection of the 3D centerlines (the views are distinct from those we match)

\section{Conclusions}

We have developed a fully automatic algorithm to perform the $3 \mathrm{D}$ reconstruction of coronary artery centerlines from three angiograms, taken from a single rotational acquisition. As demonstrated in the previous section, this approach can be used to recover the geometry of the main arteries. This is useful for determining geometrically exact 3D reconstructions of IVUS volumes ([10]). A limitation of the current algorithm is the need for breath-hold acquisitions. Approaches derived from computer vision ([11]) may help to solve this issue. The idea is to simultaneously apply the matching strategy described above and to optimize a criterion, which depends on the quality of the geometric model of the acquisitions. Another extension of this work is the application of temporal tracking algorithms ([4]) to the other frames of the rotational acquisition. The obtained results will 
then be a 4D description of the vessels as used in [5]. This model will be obtained with a single injection of contrast media and on a single plane acquisition system.

\section{Acknowledgements}

We would like to thank Guy Shechter for very helpful and enriching discussions. Our gratitude also goes to the ChIR team members (http://www-sop.inria.fr/chir/) for their everyday scientific support and friendship.

\section{References}

[1] Messenger J.C., Chen S.Y., Carroll J.D., Burchenal J.E., Kioussopoulos K. and Groves B.M., "3D coronary reconstruction from routine single-plane coronary angiograms: clinical validation and quantitative analysis of the right coronary artery in 100 patients", The International Journal of Cardiac Imaging 16(6): 413-427, December 2000.

[2] Chen S.Y. and Carroll J.D., "3-D reconstruction of coronary arterial tree to optimize angiographic visualization", IEEE Transactions in Medical Imaging 19(4), April 2000.

[3] Chen S.Y. and Carroll J.D., "Computer Assisted Coronary Intervention by Use of On-line 3D Reconstruction and Optimal View Strategy"', Medical Image Computing and Computer-Assisted Intervention Proceedings, Lecture Notes in Computer Science Vol. 1496: 377-385, Springer, Cambridge, October 1998.

[4] Shechter G., Devernay F., Coste-Manière E. and McVeigh E., "Temporal tracking of 3D coronary arteries in projection angiograms", Proceedings of SPIE Medical Imaging 4684, San Diego, February 2002.

[5] Ding Z. and Friedman M.H., "Quantification of 3-D coronary arterial motion using clinical biplane cineangiograms", The International Journal of Cardiac Imaging 16(5): 331-346, October 2000.

[6] Krissian K., Malandain G., Ayache, N., Vaillant R. and Trousset Y., "Model-Based Multiscale Detection of 3D Vessels", Proceedings of the IEEE Conference on Computer Vision and Pattern Recognition: 722-727, Santa Barbara, 1998

[7] Mourgues F., Devernay F., Malandain G. and Coste-Manière E., "3D+t Modeling of Coronary Artery Tree from Standard Non Simultaneous Angiograms”, Medical Image Computing and Computer-Assisted Intervention Proceedings, Lecture Notes in Computer Science Vol. 2208:, Springer, Utrecht, 2001.

[8] Faugeras O. and Robert L., "What can two images tell us about a third one?", European Conference on Computer Vision, 485-492, 1994.

[9] Ayache N., "Artificial Vision for Mobile Robots: Stereo Vision and Multisensory Perception”, 135-154, The MIT Press, Cambridge, 1991.

[10] Reiber J., Koning G., Dijkstra J., Wahle A., Goedhart B., Sheehan F.H. etal., "Angiography and Intravascular Ultrasound", in: Sonka M., Fitzpatrick J.M., editors. Handbook of Medical Imaging - Volume 2: Medical Image Processing and Analysis. Belligham, WA.: SPIE, 2000: 711-808.

[11] Triggs B., McLauchlan P., Hartley R., Fitzgibbon A., "Bundle Adjustment A Modern Synthesis" in "Vision Algorithms: Theory and Practice": 298-375, Springer Verlag, Lectures Notes in Computer Science, 2000. 